\title{
Self-reported cigarette smoking status imprecisely quantifies exposure in pregnancy
}

\author{
Carolina C. Venditti ${ }^{1}$, Graeme N. Smith ${ }^{1,2}$ \\ ${ }^{1}$ Department of Biomedical and Molecular Sciences, Queen’s University, Kingston, Canada \\ ${ }^{2}$ Department of Obstetrics and Gynecology, Kingston General Hospital, Kingston, Canada \\ Email: gns@queensu.ca
}

Received 28 November 2011; revised 29 December 2011; accepted 15 January 2012

\begin{abstract}
Objective: We sought to determine the validity of self-reported smoking activity versus two quantitative measurements of tobacco exposure in pregnancy. We hypothesized that pregnant women would under-report their daily smoking amounts, due to the negative social stigmas associated with such a behavior. Methods: Cigarette-smoking and non-smoking pregnant women were recruited as part of a larger research study. Pregnant women with a singleton baby $(>24$ weeks) were recruited at a clinical appointment or prior to an elective caesarian section. Self-reported smoking status, including time since last cigarette, was recorded. End-tidal breath carbon monoxide (ETCO) levels and urine cotinine levels were measured and compared. Results: Both normotensive nonsmoking $(\mathrm{NTN})(\mathrm{n}=44)$ and normotensive smoking (NTS) $(n=24)$ pregnant women were recruited. A strong correlation was found between ETCO levels and urine cotinine measurements $(r=0.6566, p<$ 0.05). Self-reported smoking status in NTS was poorly correlated with ETCO levels $(r=0.5356, p<0.05)$ and urine cotinine levels $(r=0.0324, p>0.05)$. Conclusion: Self-reported smoking status accurately identifies women who smoke in pregnancy, but not their level of tobacco exposure. Urine cotinine or ETCO are much better quantitative measurements of nicotine and carbon monoxide, respectively, and should be measured for a more precise indicator of smoking activity. These devices will allow for better counseling and monitoring of women who are trying to quit smoking and/or who enter into smoking cessation programs.
\end{abstract}

Keywords: Pregnancy; Cigarette Smoking; Carbon Monoxide; Cotinine; Self-Reported Levels

\section{INTRODUCTION}

In Canada $17 \%$ of women smoked during their pregnan-

\footnotetext{
"Self-reported smoking in pregnancy.
}

cies from 1995-2001 [1], despite the many adverse consequences, such as premature birth, low birth weight and cognitive abnormalities, that have been linked with maternal smoking [2]. Pregnancy seems to be the perfect opportunity for clinicians to offer anti-smoking advice, as women are frequently returning for appointments, and can be monitored regularly. Many clinicians rely on patient reporting to estimate tobacco exposure [3-6], however these reports are subject to error due to the possibility of false reporting [7-9], second-hand exposure, an accelerated metabolism of cigarette by-products in pregnancy, and inconsistent smoking habits [10]. Further, quantifying smoking activity has become increasingly difficult as the number of cigarette brands, sizes and strength in toxicity have amplified in the last decade.

It can be extremely difficult to counsel patients on their smoking habits due to under-reporting or nondisclosure. McClure reported that the use of a biochemical method to validate smoking habits can more effectively motivate women to stop smoking in pregnancy [11]. Uses of biochemical markers of cigarette smoke absorption, such as the nicotine metabolite cotinine and exhaled end-tidal carbon monoxide (ETCO) levels have been suggested as more accurate measures than questionnaires, due to the objectivity of their measurements [7].

Cotinine is a major metabolite of nicotine and possesses a longer half-life (19 hrs vs. 2 hrs, respectively) $[12,13]$. Found in urine, saliva, serum and plasma [7], cotinine is considered the marker of choice for objectively classifying tobacco smoking patients; its source is from nicotine metabolization [14]. There are a number of methods available to measure cotinine: colorimetric, chromatography and immunological [14]. Assay variability exists, however, and assay-specific cut-off values are necessary to differentiate smoking and non-smoking persons [7].

Carbon monoxide (CO) is a major combustible product in cigarette smoke which binds rapidly to hemoglobin forming carboxyhemoglobin (COHb). The dissociation of $\mathrm{COHb}$ occurs at the lungs, where $\mathrm{CO}$ is exhaled. 
Measuring the level of exhaled CO is an indicator of the subject's level of smoking, and it is well correlated with COHb levels $[15,16]$ also shown in our laboratory with pregnant women (Venditti and Smith, unpublished data). The half-life of CO is 3 - 4 hrs, therefore this test is dependent on the time elapsed since last cigarette smoked, and is less reliable for low-level smoking [17]. Environmental sources of CO can contribute to a person's ETCO levels [7]; although this is usually quite small and negligible compared to a smoker's CO levels. Due to its simple, quick and non-invasive fashion [15], the ETCO measuring tool is a suitable device to use in a clinical setting.

As part of a larger research study, we recruited smoking and non-smoking pregnant women and evaluated two questions. Firstly, do pregnant women accurately report themselves as smokers or non-smokers and secondly, for those who do smoke, is self-reported smoking level accurately quantified? Using two well-known measurement tools of tobacco smoke exposure, we sought to determine if reported levels of smoking correlated accurately with quantitative assessment. We hypothesized that women who smoke during pregnancy would accurately selfidentify their smoking status, but would under-report their smoking levels, and therefore would not correlate well with either of the biochemical markers of tobacco exposure.

\section{METHODS}

\subsection{Patient Recruitment and Selection}

This research study was approved by Queen's University research ethics board (OBGY-165-06). Patients were recruited by research personnel prior to an elective caesarean section, during an inpatient stay or during a clinical visit with their obstetrician. Pregnant women (>24 weeks gestation) with a singleton baby were recruited into the study and classified as either normotensive cigarette-smoking (NTS) or normotensive nonsmoking (NTN).

\subsection{Sample Collection}

Following recruitment, each mother was interviewed and data was collected regarding smoking habits. All NTS subjects were asked about second-hand smoke exposure, as well as their smoking activity: 1) Number of cigarettes smoked per day; 2) Length of time since last cigarette; 3) Number of years having smoked. A urine sample and an ETCO breath sample were then collected from each patient.

1) Urine collection

Urine $(>10 \mathrm{ml})$ was obtained for measurement of cotinine levels. All urine samples were stored in sterile plastic containers at $-80^{\circ} \mathrm{C}$ until assayed, to prevent bac- terial degradation; previously shown to degrade minimally when stored this way in excess of a year [18]. Samples were analyzed using a solid phase Cotinine enzyme-linked immunosorbent assay (ELISA) test (Calbiotech, CA, USA).

2) Expiratory End-Tidal Breath $\mathrm{CO}$

ETCO was measured using a portable automated breath analyzer (Bedfont scientific, England). The machine's methodology was previously explained by Vreman et al. [19]. Briefly, each patient was asked to hold their breath for 15 seconds and subsequently exhale completely into a disposable cardboard tube on the end of the CO breathalyzer. An automated CO level in parts per million (ppm) was calculated by the electronic device. The sensitivity and specificity of this device has been previously reported in pregnant women, with a cut off value set at $5 \mathrm{ppm}$ to differentiate smokers and nonsmokers [20]. Using the reported time since last cigarette, we corrected the ETCO levels of each subject, assuming a $3 \mathrm{hr}$ CO half-life [21]. These corrected values are used in all analyses.

\subsection{Data Analysis}

Data was analyzed using a D’Agostino and Pearson omnibus normality test for Gaussian distribution. Pearson product moment correlation (r), was used to describe the relative strength of the linear relationship between variables, and statistical significance was set at $\mathrm{p}<0.05$. We analyzed correlation coefficients with 1) both NTN and NTS and 2) only NTS data, to compare the difference between groups.

\section{RESULTS}

This study included 68 patients, 44 NTN and 24 NTS pregnant women with a mean gestational age $( \pm S D)$ at the time of recruitment of NTN: 35.1 ( \pm 3.03$)$, NTS: $32.52( \pm 5.11)$ and a mean maternal age $( \pm S D)$ of $N T N$ : $29.6( \pm 4.93)$, NTS: $27.8( \pm 5.8)$. All data sets were found to be normally distributed using the D'Agostino and Pearson Omnibus test.

All patients who smoked during pregnancy, when asked, correctly identified themselves as smokers. All nonsmoking pregnant woman also correctly identified themselves as non-smokers. All NTS subjects revealed that they were exposed to second-hand smoke on a regular basis. Data about smoking exposure and smoking habits is included in Table 1(a) for NTS volunteers.

Urine cotinine and ETCO levels were measured for both NTS and NTN volunteers, and a strong correlation was found between the two measurements $(r=0.6566$, $p$ $<0.05$ ), Figure 1. Mean urine cotinine and ETCO levels for NTS and NTN volunteers is presented in Table 1(b). 
Table 1. Comparison of volunteer smoking questionnaire data (a) and mean urine cotinine and end-tidal breath carbon monoxide (CO) for normotensive non-smokers (NTN) and smokers (NTS) (b).

\begin{tabular}{cccc}
\hline (a) & $\begin{array}{c}\text { Elapsed Time Since } \\
\text { Last Cigarette (hours) }\end{array}$ & $\begin{array}{c}\text { Years Having } \\
\text { Smoked Cigarettes }\end{array}$ & $\begin{array}{c}\text { Number of Cigarettes } \\
\text { Smoked per Day }\end{array}$ \\
\hline $\begin{array}{c}\text { Mean } \\
( \pm \text { SD) }\end{array}$ & 1.85 & 9.32 & 10.29 \\
$( \pm 2.38)$ & $( \pm 5.20)$ & $4-20$ \\
Range & $0.16-4$ & $0.67-20$ & \\
\hline (b) & $\begin{array}{c}\text { Mean Urine } \\
\text { Cotinine (SD) (ng/ml) }\end{array}$ & $\begin{array}{c}\text { Mean End-Tidal } \\
\text { Breath CO (SD) (ppm) }\end{array}$ \\
\hline NTS & $12125(8871)$ & $15.74(7.78)$ & \\
NTN & $0.95(2.75)$ & $1.84(0.91)$ & \\
\hline
\end{tabular}

In Figure 2, we present the correlation of reported cigarettes smoked per day (NTN and NTS) with ETCO (Figure 2(a)) and urine cotinine (Figure 2(b)). When analyzed with both NTN and NTS, the reported number of cigarettes smoked per day was significantly correlated with ETCO breath sample $(r=0.8728, p<0.05)$. The correlation, however, was much lower, but still significant, when analyzing only NTS data $(r=0.5356, \mathrm{p}<$ 0.05). In Figure 2(b), reported number of cigarettes smoked per day (NTS and NTN) was significantly correlated with urine cotinine measurements $(r=0.6690, p<$ $0.05)$. When analyzed using only NTS data, this correlation was not significant $(r=0.0324, \mathrm{p}>0.05)$.

\section{DISCUSSION}

The current study sought to evaluate the validity of selfreported smoking quantification among pregnant women. In addition to questionnaire data, we used two wellknown and non-invasive tools, urine cotinine analysis and an ETCO analyzer to evaluate tobacco exposure through indirect quantitative measurement.

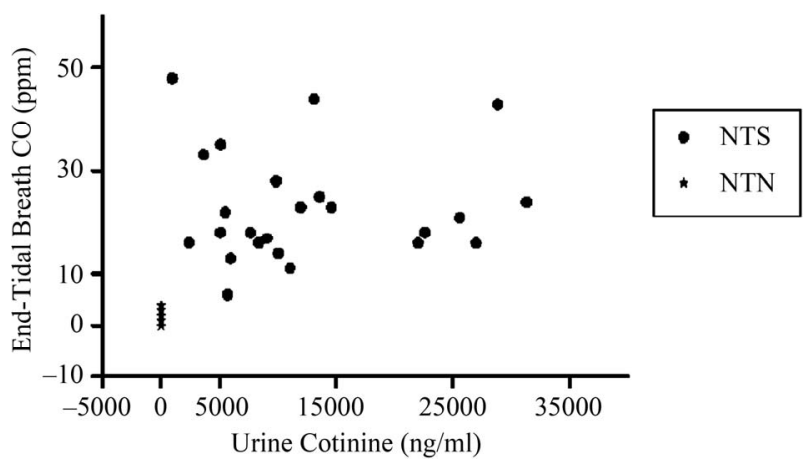

Figure 1. Maternal urine cotinine concentrations versus endtidal breath carbon monoxide (ETCO) levels. A correlation between ETCO levels and urine cotinine was computed for all volunteers, normotensive non-smoking (NTN) and smoking (NTS), with a Pearson product-moment correlation coefficient of $\mathrm{r}=$ $0.6566(\mathrm{p}<0.05)$.

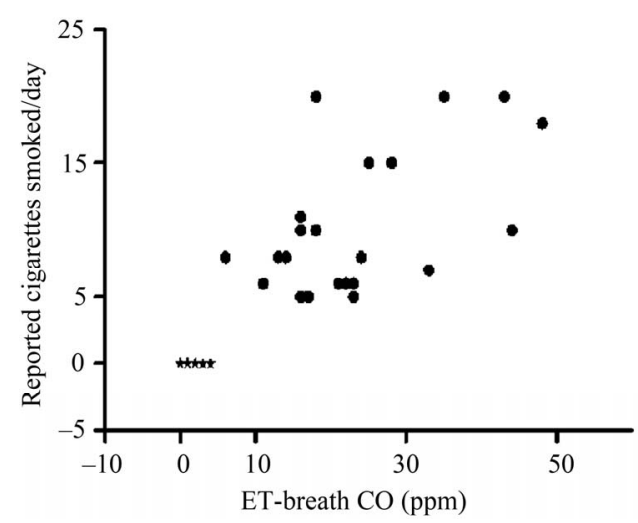

- NTS

* NTN

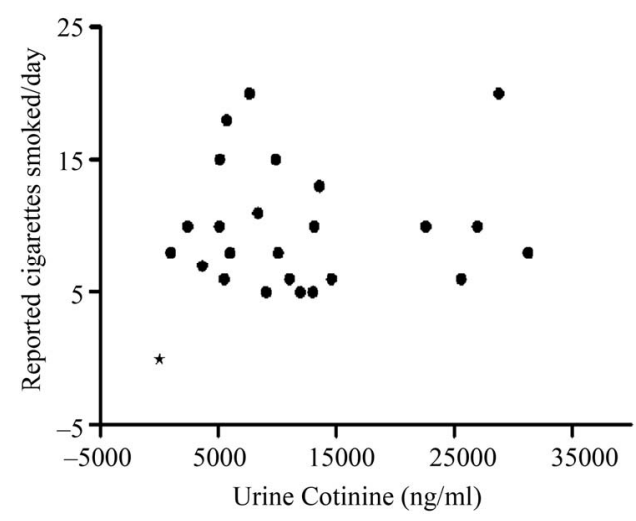

(b)

Figure 2. Reported cigarettes smoked per day versus urine cotinine and end-tidal breath CO levels. A Pearson product moment correlation was computed between reported cigarettes smoked per day and breath ETCO levels (a) normotensive nonsmoking (NTN) and smoking (NTS) $r=0.8728(\mathrm{p}<0.05)$ and only NTS $r=0.5356(p<0.05)$. Correlation coefficients were determined between reported cigarettes smoked per day and urine cotinine levels (b) NTN and NTS $r=0.6690(p<0.05)$ and only NTS $r=0.0324(p>0.05)$.

As previously reported, non-smokers should have cotinine ELISA levels below $200 \mathrm{ng} / \mathrm{ml}$ [7]; all reported non-smokers were well below this level and reported 
smokers well above. In accordance with Usmani and colleagues [20], these findings indicate that women who smoke during pregnancy are willing to reveal this information to their physician accurately. The level of smoking status, however, proved to be more complicated to evaluate, and NTS self-reported smoking quantification was not consistent with either of the biochemical tests.

The strong correlation between the ETCO samples and the urine cotinine levels $(r=0.6566, p<0.05)$, indicated that the two quantitative measurements were in accordance with one another. As studies have previously reported on each of their accuracies [19,22-24] this outcome was not surprising. Previous studies have used the Bedfont breathalyzer to measure levels of CO in NTS and NTN volunteers [25]. A concern for this test is the time frame for analysis relative to the volunteer's last cigarette; $\mathrm{CO}$ in the body has a half-life of three to four hours [21]. In our study, the mean elapsed time since last cigarette smoked was 1.85 hours, with the longest reported elapsed time being 4 hours. We also corrected for the reported time since the volunteer's last cigarette. Therefore, the recorded ETCO levels are believed to be as accurate as possible for each subject.

The correlation coefficients between self-reported smoking status and urine cotinine values $(r=0.6690, p<0.05)$ as well as with ETCO levels $(r=0.8728, \mathrm{p}<0.05)$ were in agreement, when analyzing data for NTS and NTN as one data group. The correlation coefficients decreased considerably for both urine cotinine $(r=0.0324, p>0.05)$ and ETCO $(r=0.5356, p<0.05)$ and when the analysis was conducted for NTS only. While urine cotinine levels and ETCO did correlate with self-reported smoking status overall, they were relatively poorly correlated in those who did admit to smoking, suggesting that women underestimate or under-report their actual use during pregnancy.

The quantitative results for urine cotinine are similar to previous studies reported in the literature $[22,26]$. In pregnancy, cotinine clearance is reported to increase to $140 \%$ non-pregnancy clearance rate [10], therefore higher than normal cotinine levels are found in the urine of smoking pregnant women. The metabolic changes in pregnancy that affect the breakdown of cotinine are unclear [10], although the half-life of cotinine is $9 \mathrm{hr}$ in pregnancy [10], compared to 17 hours in the non-pregnant population [13]. Jhun et al., found no significant correlation between urine cotinine and self-reported smoking habits in a smoking, pregnant population [27]. It has been shown that urine cotinine levels correlate more accurately with birth outcomes than do self-reported smoking status [28]. In accordance with our study, Klebanoff and colleagues showed that pregnant women accurately report themselves as smokers, but urine cotinine proved a more accurate marker of the level of to- bacco exposure [28].

The ETCO measurements, resulted in a low correlation coefficient with NTS self-reported smoking status, but this value was significant. Due to the strong correlation with urine cotinine values, one would expect both tests to correlate similarly with NTS self-reported smoking status. However, the ETCO test does not measure nicotine-only exposure, as does the urine cotinine test. Other combustible products such as marijuana [29], environmental $\mathrm{CO}$ and fetal/maternal endogenous $\mathrm{CO}$ production may increase the ETCO reading [7]. The breathalyzer is known to be less accurate at lower scale values [17], and with so many non-smoking volunteers in our study, this may have skewed the results slightly.

In the general public, false reporting of smoking habits is usually low [30-32]. The findings of our study, however, are based on a pregnant population, and the results in such a group are quite different. A study conducted in New Zealand found that $22 \%$ of pregnant women biochemically confirmed as smokers, when questioned, denied it. Further, there was a discrepancy between reported smoking amount and objective measure in $12 \%$ of the population [33]. Patrick et al. explains that smoking reports are generally accurate, but with intensity of antismoking environment comes an increase in false-reporting of smoking status. They found that in pregnancy, up to $25 \%$ of smokers falsely declare themselves as nonsmokers [30], while another group found that pregnant smokers may declare a reduced consumption of smoking [34].

Ultimately, the correlation between urine cotinine and ETCO levels was better than that of each test with NTS reported cigarette-smoking amount. This may be due to the generalized reports of smoking levels in clumps of 5 , 10,15 or 20 cigarettes per day, or $1 / 4,1 / 2,3 / 4$ pack per day; or as others have reported, it may be due to the different smoking puff patterns between individuals, yielding more or less nicotine/cigarette [17,35]. Although the number of cigarettes smoked may seem to be under-reported in the pregnant population, it is impossible to determine puff length (length of time one inhales on a cigarette per breath) per cigarette; cigarette number may decrease, but puff length may increase, therefore maintaining or even increasing nicotine exposure [36]. Additionally, the actual amount of a cigarette each subject smoked could be variable. Therefore, women may not be under-reporting the number of cigarettes smoked per day, but rather correcting for the decreased nicotine intake, by puffing longer and more strongly on each cigarette. In this way, women would maintain their nicotine levels (biochemical markers), and the biochemical tests then, would not correlate well with self-reported smoking status.

There are limitations to this study. Specifically, women 
were not questioned about their use of other sources of nicotine, namely a nicotine patch. This may have added variability when measuring the urine cotinine levels, and may have contributed to the non-smokers who measured small amounts of cotinine in their urine. While all women who identified themselves as smokers also confided that they were regularly around second hand smoke, non-smokers were not questioned on this information and this may explain the eight NTN women with ETCO levels at 3 and $4 \mathrm{ppm}$, and may have impacted on the NTN urine cotinine levels (though in reality they were all below the cut off levels for smokers). Finally, we did not question NTN or NTS women about other tobacco exposures such as chewing tobacco.

Our findings have implications for clinical practice, as well as clinical studies where self-reported smoking status is used to comment on the causes and effects of smoking. Using objective, quantitative tests allows physicians and researchers a further method of verifying smoking activity, as well as a more convincing tool to offer smoking cessation advice. In addition, these tools could be used to monitor smoking cessation programs for pregnant women.

\section{CONCLUSION}

We believe self-reporting questionnaires are appropriate for use in categorizing pregnant smokers and non-smokers, as women tend to accurately identify themselves into each of the categories [20,34]. The problem lies in the amount of cigarette smoking, and therefore tobacco exposure, that women disclose to their physician. In this study, we showed that both quantitative tests strongly correlated with one another, but did not match the selfreported smoking levels reported by the NTS patients. As a stronger indication of tobacco exposure, the urine cotinine test is a precise and minimally invasive technique to achieve reliable quantitative results. However, in view of the time constraints of a clinical setting, we suggest the use of the ETCO breathalyzer as a rapid measurement tool, where immediate results can be used to further counsel patients and offer anti-smoking advice. This device is currently used in Scotland with all pre partum patients [20]. Women whose ETCO is above 5 ppm are all referred to smoking cessation support programs. We hope that physicians will consider using this device as an additional means of counseling patients about smoking during pregnancy.

\section{REFERENCES}

[1] Miller, W.J. and Hill, G. (2011) Pregnancy and smoking. Health Reports, 53-56.

[2] Werler, M.M., Pober, B.R. and Holmes, L.B. (1985)
Smoking and pregnancy. Teratology, 32, 473-481. doi:10.1002/tera.1420320316

[3] Lindqvist, P.G. and Marsal, K. (1999) Moderate smoking during pregnancy is associated with a reduced risk of preeclampsia. Acta Obstetricia et Gynecologica Scand, 78, 693-697. doi:10.1080/j.1600-0412.1999.780806.x

[4] Zhang, J., Klebanoff, M.A., Levine, R.J., Puri, M. and Moyer, P. (1999) The puzzling association between smoking and hypertension during pregnancy. American Journal of Obstetrics \& Gynecology, 181, 1407-1413. doi:10.1016/S0002-9378(99)70384-4

[5] Soothill, P.W., Morafa, W., Ayida, G.A. and Rodeck, C.H. (1996) Maternal smoking and fetal carboxyhaemoglobin and blood gas levels. British Journal of Obstetrics and Gynaecology, 103, 78-82.

doi:10.1111/j.1471-0528.1996.tb09519.x

[6] Duffus, G.M. and MacGillivray, I. (1968) The incidence of pre-eclamptic toxaemia in smokers and non-smokers. Lancet, 1, 994-995. doi:10.1016/S0140-6736(68)91106-9

[7] Jarvis, M.J., Tunstall-Pedoe, H., Feyerabend, C., Vesey, C. and Saloojee, Y. (1987) Comparison of tests used to distinguish smokers from nonsmokers. American Journal of Public Health, 77, 1435-1438. doi:10.2105/AJPH.77.11.1435

[8] Sillett, R.W., Wilson, M.B., Malcolm, R.E. and Ball, K.P. (1978) Deception among smokers. British Medical Journal, 2, 1185-1186. doi:10.1136/bmj.2.6146.1185

[9] Ohlin, P., Lundh, B. and Westling, H. (1976) Carbon monoxide blood levels and reported cessation of smoking. Psychopharmacology, 49, 263-265. doi:10.1007/BF00426827

[10] Dempsey, D., Jacob, P. III and Benowitz, N.L. (2002) Accelerated metabolism of nicotine and cotinine in pregnant smokers. Journal of Pharmacology and Experimental Therapeutics, 301, 594-598. doi:10.1124/jpet.301.2.594

[11] McClure, J.B. (2004) Motivating prepartum smoking cessation: A consideration of biomarker feedback. Nicotine \& Tobacco Research, 6, S153. doi:10.1080/14622200410001669222

[12] Willers, S., Skarping, G., Dalene, M. and Skerfving, S. (1995) Urinary cotinine in children and adults during and after semiexperimental exposure to environmental tobacco smoke. Archives of Environmental Health, 50, 130-138. doi:10.1080/00039896.1995.9940890

[13] Benowitz, N.L., Kuyt, F., Jacob, P., Jones, R.T. and Osman, A.L. (1983) Cotinine disposition and effects. Clinical Pharmacology \& Therapeutics, 34, 604-611. doi:10.1038/clpt.1983.222

[14] Haufroid, V. and Lison, D. (1998) Urinary cotinine as a tobacco-smoke exposure index: A minireview. International Archives of Occupational and Environmental Health, 71, 162-168. doi:10.1007/s004200050266

[15] Jarvis, M.J., Russell, M.A. and Saloojee, Y. (1980) Expired air carbon monoxide: A simple breath test of tobacco smoke intake. British Medical Journal, 281, 484485. doi:10.1136/bmj.281.6238.484

[16] Stevenson, D.K., Vreman, H.J., Oh, W., et al. (1994) 
Bilirubin production in healthy term infants as measured by carbon monoxide in breath. Clinical Chemistry, 40, 1934-1939.

[17] Frederiksen, L.W. and Martin, J.E. (1979) Carbon monoxide and smoking behavior. Addictive Behaviors, 4, 2130. doi:10.1016/0306-4603(79)90017-0

[18] Watts, R.R., Langone, J.J., Knight, G.J. and Lewtas, J. (1990) Cotinine analytical workshop report: Consideration of analytical methods for determining cotinine in human body fluids as a measure of passive exposure to tobacco smoke. Environmental Health Perspectives, 84, 173-182.

[19] Vreman, H.J., Stevenson, D.K., Oh, W., et al. (1994) Semiportable electrochemical instrument for determining carbon monoxide in breath. Clinical Chemistry, 40, 19271933.

[20] Usmani, Z.C., Craig, P., Shipton, D. and Tappin, D. (2008) Comparison of CO breath testing and women's self-reporting of smoking behaviour for identifying smoking during pregnancy. Substance Abuse Treatment, Prevention, and Policy, 3, 4. doi:10.1186/1747-597X-3-4

[21] Bengea, S., Araki, Y., Ito, O., Igarashi, J., Sagami, I. and Shimizu, T. (2004) Analysis of the kinetics of CO binding to neuronal nitric oxide synthase by flash photolysis: Dual effects of substrates, inhibitors, and tetrahydrobiopterin. Journal of Inorganic Biochemistry, 98, 1210-1216. doi:10.1016/j.jinorgbio.2004.04.009

[22] Vine, M.F., Hulka, B.S., Margolin, B.H., Truong, Y.K., et al. (1993) Cotinine concentrations in semen, urine, and blood of smokers and nonsmokers. American Journal of Public Health, 83, 1335-1338. doi:10.2105/AJPH.83.9.1335

[23] Seidman, D.S., Paz, I., Merlet-Aharoni, I., Vreman, H., Stevenson, D.K. and Gale, R. (1999) Noninvasive validation of tobacco smoke exposure in late pregnancy using end-tidal carbon monoxide measurements. Journal of Perinatology, 19, 358-361. doi:10.1038/sj.jp.7200193

[24] Kreiser, D., Baum, M., et al. (2004) End tidal carbon monoxide levels are lower in women with gestational hypertension and pre-eclampsia. Journal of Perinatology, 24, 213-217. doi:10.1038/sj.jp.7211062

[25] Irving, J.M., Clark, E.C., Crombie, I.K. and Smith, W.C. (1988) Evaluation of a portable measure of expired-air carbon monoxide. Preventive Medicine, 17, 109-115. doi:10.1016/0091-7435(88)90076-X

[26] Jordanov, J.S. (1990) Cotinine concentrations in amniotic fluid and urine of smoking, passive smoking and nonsmoking pregnant women at term and in the urine of their neonates on 1st day of life. European Journal of Pediatrics, 149, 734-737.

\section{doi:10.1007/BF01959534}

[27] Jhun, H.J., Seo, H.G., Lee, D.H., et al. (2010) Self-reported smoking and urinary cotinine levels among pregnant women in Korea and factors associated with smoking during pregnancy. Journal of Korean Medical Science, 25, 752-757. doi:10.3346/jkms.2010.25.5.752

[28] Klebanoff, M.A., Levine, R.J., Clemens, J.D., DerSimonian, R. and Wilkins, D.G. (1998) Serum cotinine concentration and self-reported smoking during pregnancy. American Journal of Epidemiology, 148, 259-262.

[29] Wu, T.C., Tashkin, D.P., Djahed, B. and Rose, J.E. (1988) Pulmonary hazards of smoking marijuana as compared with tobacco. The New England Journal of Medicine, 318, 347-351. doi:10.1056/NEJM198802113180603

[30] Patrick, D.L., Cheadle, A., Thompson, D.C., Diehr, P., Koepsell, T. and Kinne, S. (1994) The validity of selfreported smoking: A review and meta-analysis. American Journal of Public Health, 84, 1086-1093. doi:10.2105/AJPH.84.7.1086

[31] Caraballo, R.S., Giovino, G.A., Pechacek, T.F. and Mowery, P.D. (2001) Factors associated with discrepancies between self-reports on cigarette smoking and measured serum cotinine levels among persons aged 17 years or older: Third National Health and Nutrition Examination Survey, 1988-1994. American Journal of Epidemiology, 153, 807-814. doi:10.1093/aje/153.8.807

[32] Vartiainen, E., Seppala, T., Lillsunde, P. and Puska, P. (2002) Validation of self reported smoking by serum cotinine measurement in a community-based study. Journal of Epidemiology and Community Health, 56, 167-170. doi:10.1136/jech.56.3.167

[33] Ford, R.P., Tappin, D.M., Schluter, P.J. and Wild, C.J. (1997) Smoking during pregnancy: How reliable are maternal self reports in New Zealand? Journal of Epidemiology and Community Health, 51, 246-251. doi:10.1136/jech.51.3.246

[34] Lindqvist, R., Lendahls, L., Tollbom, O., Aberg, H. and Hakansson, A. (2002) Smoking during pregnancy: Comparison of self-reports and cotinine levels in 496 women. Acta Obstetricia et Gynecologica Scand, 81, 240-244. doi:10.1034/j.1600-0412.2002.810309.x

[35] Herning, R.I., Jones, R.T., Bachman, J. and Mines, A.H. (1981) Puff volume increases when low-nicotine cigarettes are smoked. British Medical Journal, 283, 187-189. doi:10.1136/bmj.283.6285.187

[36] Benowitz, N.L., Hall, S.M., Herning, R.I., Jacob, P., Jones, R.T. and Osman, A.L. (1983) Smokers of lowyield cigarettes do not consume less nicotine. The New England Journal of Medicine, 309, 139-142. doi:10.1056/NEJM198307213090303 